\title{
CONTRIBUTION A L'ÉTUDE DES LEUCOCYTES DU LAIT ET ESSAI DE LEUCOCYTO-DIAGNOSTIC DES DIFFÉRENTS ÉTATS PHYSIOLOGIQUES ET PATHOLOGIQUES DE CETTE SÉCRÉTION
}

\author{
par E. BOURGEOIS \\ Docteur-Vétérinaire \\ Vétérinaire inspecteur sanitaire du Département de la Seine
}

(Suite)

\section{Etablissement de la formule leucocytaire des laits}

Examinons successivement les variations de la formule leucocytaire selon les cas : laits normaux, laits de rétention, colostrum et laits de mammites ou plus généralement laits infectés. Pour classer ainsi les laits prélevés, nous avons fait appel à nos connaissances cliniques et anatomopathologiques et à différents procédés d'examen du lait : a) observation de ses caractères objectifs, $b$ ) centrifugation, $c$ ) examen microscopique au point de vue microbien et enfin $d$ ) détermination du degré de catalase (1). Ce n'est qu'après nous être fait uneidée d'un échantillon de lait

(1) Détermination du degré de catalase. - Au cours des pages qui vont suivre, nous traduirons le degré de catalase des laits examinés en centimètres cubes d'oxygène dégagé; aussi tenons-nous à donner quelques précisions au sujet de l'obtention de ces ehiffres.

La eatalase, diastase trouvée en abondance dans les boues de centrifugation des laits est considérée par certains auteurs comme un produit d'origine leucocytaire ; pour d'autres, au contraire, les microbes seraient les grands producteurs de catalase. Quoi gu'il en soit, les laits très riches en leucoeytes et en microbes sont toujour caxвactérisés par un degré catalasimétrique élevé.

quant un dégagement d'oxygène moléculaire, il suffira d'évaluer le volume d'oxygène dégagé, après addition d'eau oxygénée à un lait dans certaines conditions, pour avoir une idée de la richesse de ce lait en catalase. Pour ce faire, nous avons adopté l'appareil de KGSTLER :

Matériel. - Godet de verre portant 3 traits de jauge à 10,15 et 25 centimètres cubes;

Bouchon de eaoutchoue percé d'un trou laissant passer un petit tube à dégagement de quelques centimètres de longueur, effilé à sa partie supérieure.

Eprouvette à gaz de 12 centimètres cubess gradué en dixièmes de centimètre cube;

Grand récipient contenant de l'eau, qui sera maintenue pendant toute la durée de l'expérience à une température de 25 degrés centigrades environ.

Conditions de l'expirience. - Dans le godet de verre, on place 10 centimètres cubes du lait à examiner, puis 5 centimètres cubes du mélange:

Eau oxygénée à 12 volumes
Eau distillée

On enfonce le bouchon jusqu'au troisième trait de jauge de telle façon que le voume d'air emprisonné entre la surface du liquide et le bouchon soit toujours le même ; l'appareil ainsi préparé est placé dans le récipient plein d'eau; le godet et le tube à dégagement doivent être complètement immergés. L'éprouvette graduée est alors retournée remplie d'eau sur l'appareil, coiffant la pointe effilée du tube de dégagement; l'ouverture de l'éprouvette ne doit pas être au contact du bouchon puisqu'il est indispensable de ménager un espace pour l'évacuation de l'eau chassée par le dégagement d'oxygène.

La lecture est faite au bout d'une heure sur l'éprouvette graduée; les ehiffres repré- 
grâce à ces différents moyens d'investigation, que nous avons procédé à l'établissement de sa formule leucocytaire, et nous pouvons dire $q u$ 'à chacun des trois grands groupes cités plus haut: laits normaux, laits de rétention ou colostrum et laits infectés, correspondent trois types très différents de formules leucocytaires.

A. Latts normaux. - Les laits classés dans cette catégorie offraient les caractéristiques suivantes : ils provenaient de vaches en bonne santé, en pleine période de lactation, subissant des traites régulières et complètes, de rendement normal. Les quartiers sur lesquels ils furent prélevés ne présentaient eliniquement rien d'anormal et les ganglions rétromammaires étaient sains. Ces laits d'aspect et de goût normaux avaient tous un faible degré de catalase correspondant à un dégagement d'oxygène de 0 à 2 centimètres cubes. Le culot de centrifugation était toujours très peu abondant, non visqueux, comme pulvérulent et se prêtait bien, après dilution, à la confection de frottis; à l'examen de ceux-ci, les leucocytes apparaissaient peu serrés et aucun germe phagocyté ne fut observé.

Nous devons à la vérité de dire que peu de laits, neuf pour cent environ, remplirent ces conditions, à dessein très sévères, de façon à ne retenir que des laits indiscutablement sains. La plupart des laits, en effet, offrirent à l'examen microscopique d'innombrables variétés de germes phagocytés (cocci, fins bacilles, bâtonnets, etc.) peut-être souvent dépourvus de rôle pathogène, mais ne les ayant pas identifiés, ces microbes nous semblèrent a priori suspects, et aucun lait présentant des germes phagocytés ne fut retenu pour l'établissement de la formule leucocytaire des laits normaux.

Le tableau VI résume les formules leucocytaires de 10 laits remplissant toutes les conditions exigées ci-dessus et pour cela considérés eomme normaux.

Ces laits peuvent être ainsi caractérisés au point de vue leucocytaire : ce qui frappe d'abord à l'examen des frottis, c'est la raretédes leucocytes. Comme dans un quartier sain, trait à fond et à intervalles réguliers, aucun appel leucocytaire ne se justifie, les leucocytes observés ne peuvent être que des éléments de filtration, partant peu nombreux.

L'établissement de la formule leucocytaire permet en outre les constatations suivantes: pourcentage relativement bas (par rapport à

sentent des centimètres cubes d'oxygène dégagé et traduiront pour nous au cours de ce travail le degré catalasimétrique des laits étudiés.

Pour déterminer en même temps le degré catalasimétrique des laits des quatre quartiers d'une mamelle, nous avons imaginé un dispositif de fortune très simple pouvant contenir quatre des appareils ci-dessus décrits. Nous recommandons toutefois la mise en place de chaque appareil aussitôt après avoir effectué le mélange de lait et d'eau oxygénée diluée, car le dégagement commence immédiatement, et, si le lait est très riche en catalase, avec une grande intensité. 
Tabieau VI

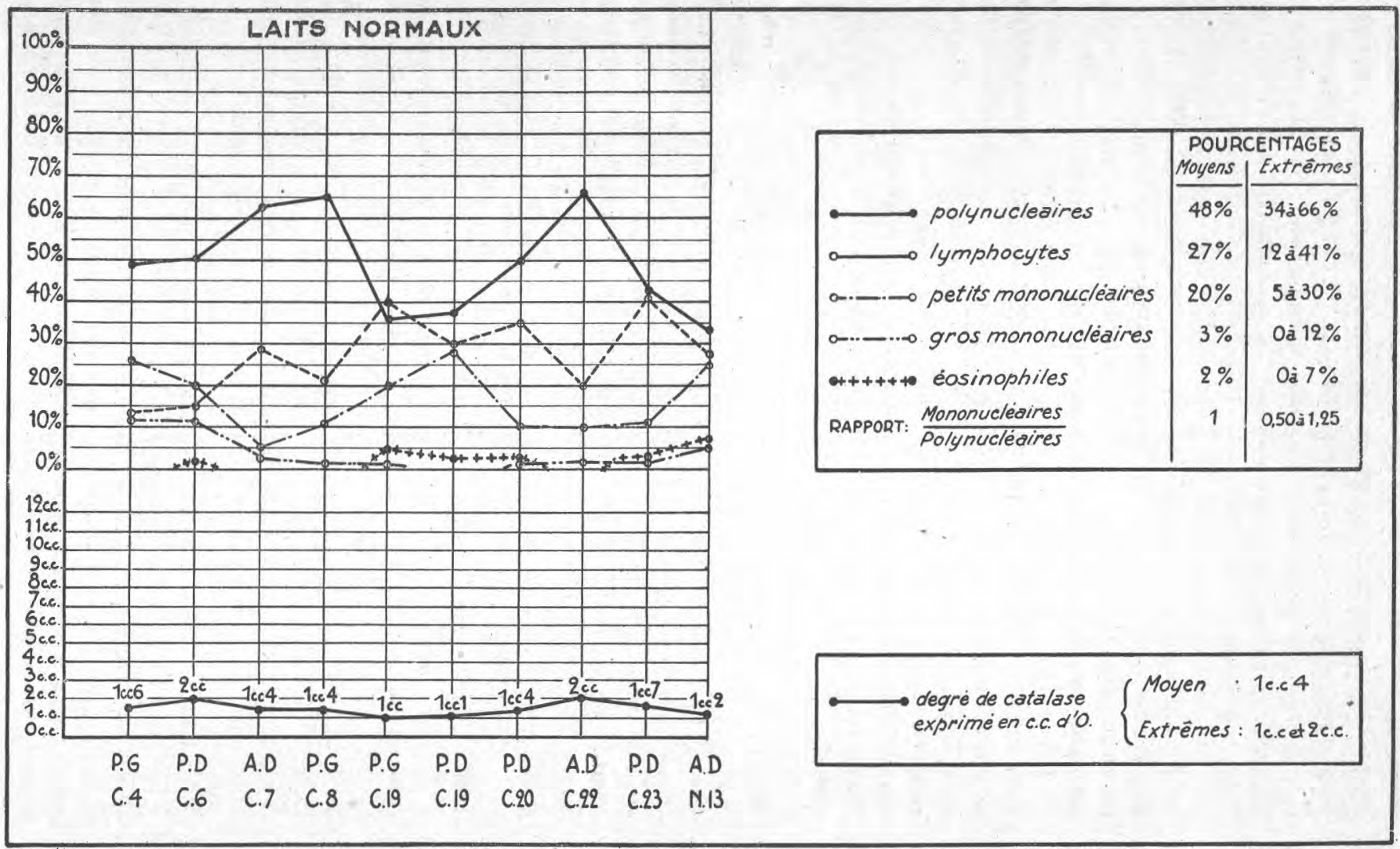


celui que nous reneontrerons dans les autres catégories de laits) de polynucléaires neutrophiles, 48 en moyenne; pourcentage important de lymphocytes, 27 en moyenne ; faible proportion des gros mononueléaires, $3 \%$, absence de mononucléaires chargés de graisse. Notons que pour les laits normaux, VARRIER Jones trouve que les lymphocytes constituent la presque totalité des leucocytes, les polynucléaires n'y étant que très rares. Nos résultats ne concordent donc pas avec les siens; mais ils ne sont pas contradietoires, car, de toutes les catégories de laits étudiées, ce sont les laits, considérés par nous comme normaux, qui ont le pourcentage le plus élevé de lymphocytes, éléments de filtration par excellence et celui le plus faible de polynucléaires.

Si nous établissons le rapport $\frac{\mathrm{M}}{\mathrm{P}} \frac{\text { (mononucléaires) }}{\text { (polynucléaires) }}$, nous voyons qu'il varie pour ces dix échantillons de lait de 0,50 à 1,25 (1 en moyenne); toutes ces observations peuvent être résumées en disant que pour les laits normaux, le rapport $\frac{\mathrm{M}}{\mathrm{P}} \mathrm{n}$ 'est jamais inférieur à 0,50 .

VARIATIONS DU CONTENU LEUCOCYTATRE DES LAITS NORMAUX SELON LE QUARTIER. - Un simple coup d'œil sur le tableau IV nous a permis d'observer, qu'au point de vue leucocytaire, les laits de chacun des quatre quartiers d'une même mamelle pouvaient n'avoir aucune similitude et nous avons vu que seul l'état microbien pouvait être responsable de cette discordance, toute rétention partielle mise à part.

Si les laits ne sont pas microbiens, ce qui est rare, il en est autrement; ainsi les laits des quartiers $\mathrm{P}$ G et $\mathrm{P}$ D de l'animal C 19, dont les formules leucocytaires sont résumées dans le tableau VI, offrent un certain rapprochement: proportion à peu près égale de polynucléaires (36 et $37 \%$ ) ; respectivement 40 et $30 \%$ de lymphocytes ; 20 et $30 \%$ de petits mononucléaires; 4 et $3 \%$ enfin d'éosinophiles. Cette unique observation de deux laits issus d'une même mamelle et réalisant les conditions sévères exigées pour qu'ils puissent être considérés comme normaux, nous permet de dire que les laits des quartiers d'une même mamelle, s'ils sont normaux, ont des formules leucocytaires très voisines. Ces formules leucocytaires sont, ainsi que nous l'avons vu dans un chapitre précédent, très proches de celles du sang de l'animal, dont elles sont le reflet, avec cette différence toutefois que les lymphoeytes, venus aussi de la lymphe, y sont en plus grande proportion.

VARIATIONS DE LA FORMULE LEUCOCYTATRE AU COURS DE LA TRATTE. - De tous les examens comparatifs des formules leucocytaires des laits d'un même quartier, prélevés au début, au milieu ou à la fin de la traite, - il résulte que les, pourcentages des diverses catégories de leucocytes sont toujours sensiblement les mêmes, les faibles différences constatées sont de l'ordre de celles qu'on peut attribuer aux irrégularités de numération; 
elles ne sont jamais susceptibles de troubler la physionomie leucocytaire du lait et par conséquent de fausser le diagnostic.

VARIATIONS DE LA FORMULE LEUCOCYTAIRE AU COURS DE LA PÉRIODE DE lactation. - Au cours de la période de lactation, deux processus, rétention et infection, influenceront la formule leucocytaire; les laits, pour lesquels l'un ou l'autre de ces deux processus ne joue pas, sont l'exception ; ce sont ceux cités plus haut et appelés à juste titre normaux. En effet la mamelle infectée eśt presque la règle; si la clinique peut déceler les infections aigües, tapageuses, elle est incapable de dénoncer les infections chroniques insidieuses; aussi combien de laits, qu'on croit à tort issus d'une mamelle saine, sont en réalité infectés ? Cette infection latente peut succéder à une infection aigüe; dans ce cas la glande après avoir été gonflée, douloureuse, durcie, peut avoir retrouvé toute sa souplesse, et le lait, après avoir été grumeleux, modifié dans sa teinte et sa viscosité, peut avoir repris son aspect normal. L'illusion de la guérison clinique fait considérer que le lait est sain, alors qu'il est toujours microbien et plus ou moins modifié dans ses caractères chimiques et biologiques. Le plus souvent, seul un rendement diminué trahit l'infection; c'est alors que le propriétaire espace davantage les traites dans le vain espoir de les voir plus abondantes et à l'infection latente viendra s'ajouter la rétention. La vidange de la glande ne s'effectuant plus assez souvent, les germes cultiveront à souhait dans les acini et l'infection s'accentuera jusqu'à redevenir à son stade primitif. Rétention et infection s'appellent réciproquement et il est rare que ces deux processus n'évoluent pas "pari passu". Ceci nous amène à penser que dans la plupart des laits, le contenu leucocytaire est qualitativement influencé par ces deux facteurs. Aussi, pour déterminer réciproquement les modifications qu'ils font subir à la formule leucocytaire, nous est-il indispensable de nous adresser à des laits de rétention prononcée peu infectés et à des laits très infectés, mais de rétention très légère. L'étude du colostrum et des mammites streptococciques nous donnera des renseignements très nets à cet égard.

B. Latts de rétention et colostrum. - Par la pression qu'il exerce sur les cellules sécrétrices dans les cas de rétention, le lait accumulé dans l'acinus est un obstacle à une nouvelle sécrétion ; l'organisme dès ce moment met en ouvre tous les moyens dont il dispose pour éliminer le lait non amené à l'extérieur et qui est devenu un corps étranger. La phagocytose est le plus efficace de ces moyens de défense; le contenu leucocytaire devient aussitôt plus abondant et l'appel leucocytaire établi a pour résultat d'éloigner la formule leucocytaire du lait de celle du sang par la prédominance d'une catégorie déterminée de leucocytes spécialisés dans cette défense particulière. Les leucocytes chargés de ce travail sont les gros mononucléaires. En 
effet, dès que la rétention s'installe dans un quartier de la mamelle, leur pourcentage peut atteindre 20 à 30 , alors que dans les laits normaux on en trouve guère que 3 à $4 \%$ en moyenne et pas du tout dans un certain nombre de cas.

Ces éléments procèdent à l'évacuation des globules gras qu'ils phagoeytent; aussi parallèlement à eux trouve-t-on dans la formule leucocytaire des mononucléaires dont le protoplasma est plus ou moins parsemé de globules parfaitement arrondis et ineolores. Nous avons chaque fois établi séparément le pourcentage de ces leucocytes devenus lipophages. Si la rétention s'accentue, ces lipophages se gorgent de globules gras au point d'apparaître sous forme d'un cercle muriforme de 15 à $20 \mu$ de diamètre dans lequel on reconnait avec beaucoup de difficulté un noyau très dégénéré ; c'est à cet aspect spéeial qu'on a donné le nom de globule du colostrum ou de corpuscule de Donnḱ.

Les auteurs ne sont pas d'accord sur l'origine de ces globules du colostrum. Beaucoup d'auteurs, VARRIER Jonks et ERnsT notamment les considèrent eneore comme des cellules mammaires. DUCLERT, BAB, Weill et Thévenet, confirmant Czerny, voient au contraire en eux des leucocytes chargés de graisse. Il semble que depuis les recherches expérimentales sur le colostrum des Professeurs Ch. Porcher et L. PAnisset, cette dernière interprétation seule devrait être admise. Citons leur important travail: "Le fonctionnement physiologique de " la cellule mammaire n'a pas deux manières différentes de s'exprimer : " il n'est pas exact de dire que la mamelle, avant le part et dans les: " quelques jours qui suivent, sécrète du colostrum; c'est du lait qu'elle: " donne, mais du lait dont nous n'avons qu'une image déformée par des "actions phacocytaires; en d'autres termes, le colostrum est un reliquat "de phagocytose d'un lait antérieurement produit."

Pour prouver leur manière de voir ces auteurs rendent compte d'une expérience très suggestive : ils injectent du lait stérile dans le péritoine d'un cobaye. Après avoir constaté la résorption de la partie liquide du lait et la disparition du lactose, ces auteurs observent un amas leucocytaire où les polynueléaires prédominent 6 à 7 heures après l'injection, alors qu'au bout de 24 heures, les mononucléaires sont les plus nombreux. Ges deux catégories de leucocytes se montrent capables d'englober les globules graisseux du lait injecté; tantôt ils les bourrent au point d'en masquer le noyau, on a alors des corpuscules de Donnḱ; de gros globules plus difficilement phagocytés circulent librement entre: les corpuscules. Les auteurs renouvellent l'expérience en injectant cette fois du lait homogénéisé : l'englobement total de la graisse est alors réalisé, les polynucléaires prédominent toujours dans ce dernier cas. Les auteurs concluent que polynucléaires et mononucléaires interviennent dans le cas de rétention, les premiers étant de préférence microphages, les seconds, dont le rôle est plus important, étant plutôt des macrophages. 
Nous adoptons entièrement le point de vue de ces auteurs en ce qui concerne la formation du colostrum et la nature des corpuscules de DoNNÉ; comment en effet admettre la présence de cellules mammaires dans le péritoine?

D'autre part, nos examens leucocytaires de laits de rétention et de colostrum confirment en tous points cette interprétation :

$1^{\circ}$ Nous avons noté un parallélisme constant entre la présence des gros mononucléaires et celles des mêmes éléments chargés de graisse;

$2^{\circ}$ Nous avons observé tous les stades intermédiaires entre les gros mononucléaires dépourvus de globules gras et les corpuscules de DonNé.

$3^{\circ}$ Dans les frottis de colostrum, nous avons trouvé des polynucléaires lipophages.

Caraotéristiques des latts de rétention. - Les échantillons de laits classés dans cette catégorie provenaient de quartiers pour lesquels la rétention était plus ou moins prononcée, selon la cause qui l'avait fait naître, ou compliquée d'infection chronique sans symptômes apparents, mais ayant déterminé un abäissement du rendement laitier avec, comme conséquence erronée, l'espacement et l'insuffisance des traites.

Etudions successivement les différents cas de rétention résumés dans la première partie du tableau VII (p. 754).

Io Rétention prononcée. - Les deux premiers échantillons étudiés furent prélevés sur la vache laitière No 2,8 ans environ, mise en vente au Marché de la Villette, réformée pour la boucherie par suite de mammite très ancienne des quartiers antérieur et postérieur droits. A cause de l'hypertrophie et de la dureté des quartiers malades, les deux autres quartiers demeurés sains n'ont pu depuis longtemps être l'objet de traites régulières, et depuis une semaine au moins, époque de la vente de l'animal au département d'origine, il est vraisemblable qu'aucune traite fut effectuée. Nous sommes donc bien ici en présence d'un cas de rétention prononcée.

Les liquides prélevés sur les quartiers antérieur et postérieur ganches, cliniquement sains, ont une viscosité augmentée et un aspect un peu jaunâtre dû à l'excès de matière grasse; en effet, après centrifugation, les deux tiers supérieurs du tube sont occupés par la crème et le tiers inférieur seulement par le lait écrémé; un petit culot, quantitativement normal et non visqueux, se prête bien, après dilution, à la confection des frottis; aucun germe phagocyté à l'examen microscopique.

$\mathrm{Si}$ on compare la formule leucocytaire de ces deux laits à celles des laits normaux du tableau VI, on voit que cette rétention prononcée se traduit par un pourcentage énorme de mononucléaires chargés de globules gras (34 et $29 \%$ ) plus important que celui, cependant anormal, des gros mononucléaires ordinaires (12 et $20 \%$ ); et cela, au détriment 
Tablead VII

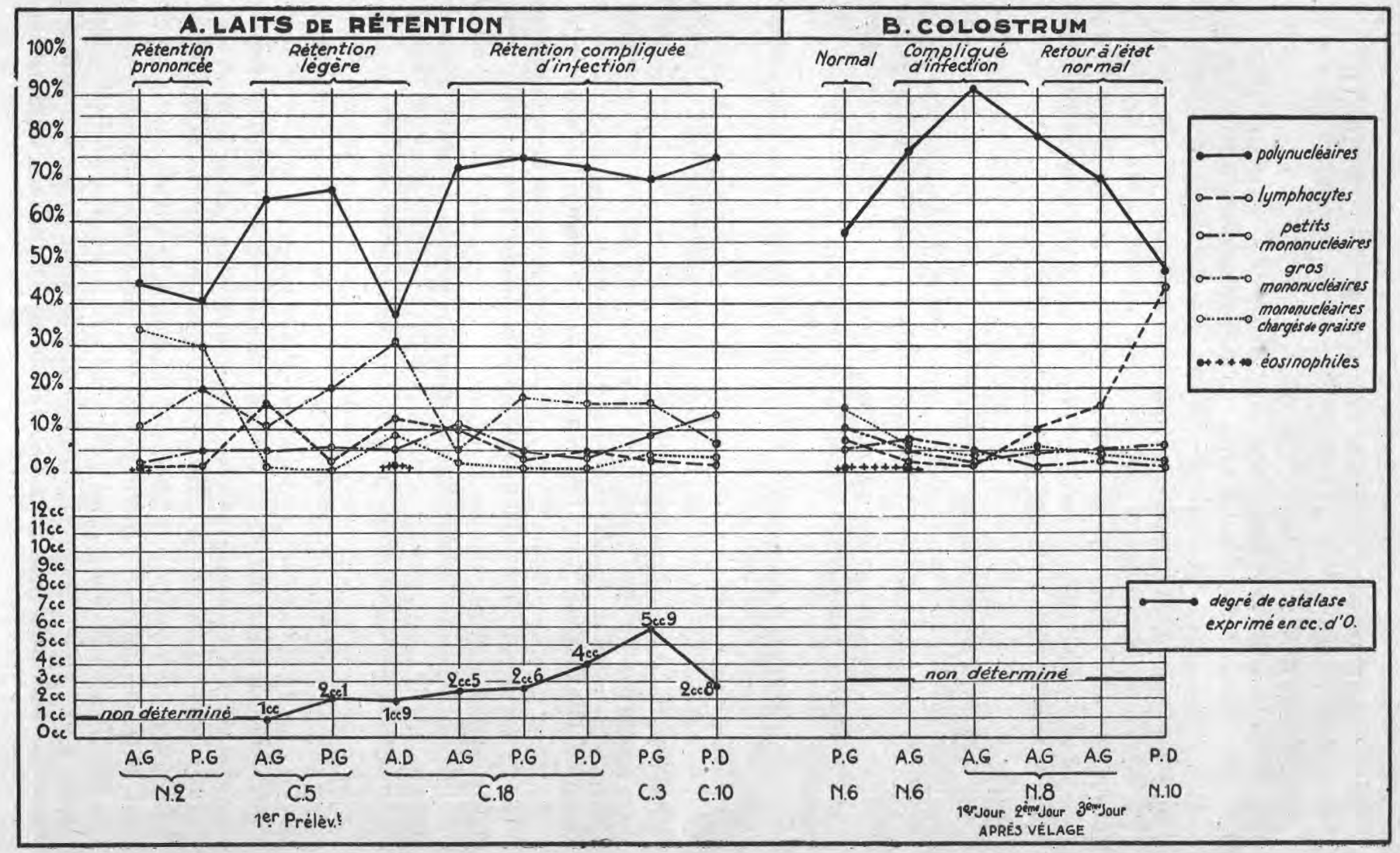


de celui très faible des lymphocytes. Notons, en outre, un pourcentage normal des polynucléaires, ce qui prouve l'absence d'infection, chose exceptionnelle en de telles circonstances.

$2^{\circ}$ Rétention légk̀Re. - Les trois échantillons, dont l'histoire est résumée sur le tableau VII, nous offrent le type de laits de rétention légère; les deux premiers (quartiers antérieur et postérieur gauches) proviennent de l'animal $\mathrm{C} 5$ au troisième mois de lactation, eraintif et pour cela difficile à traire; le troisième, prélevé sur le quartier antérieur droit de l'animal $\mathrm{C} 18$ une dizaine de jours après le vêlage, avait été l'objet de traites insuffisantes.

Ces laits ne présentaient rien d'anormal ni dans leur aspect, ni dans leur viscosité, ni dans leur culot de centrifugation; matière grasse abondante, aucun germe phagocyté. Si nous comparons leurs formules leucocytaires à celles des laits normaux du tableau VI, nous verrons qu'elles diffèrent seulement par le pourcentage élevé des gros mononucléaires $(11 \%, 20 \%$ et $31 \%)$ et par l'apparition de mononucléaires chargés de graisse $(1 \%, 2 \%$ et $9 \%)$; cette augmentation s'est faite au détriment des lymphocytes. Remarquons, en outre, que lorsque la rétention est légère, le pourcentage des gros mononucléaires est supérieur à celui des mêmes éléments chargés de graisse; le contraire a été constaté dans les cas de rétention prononcée.

Il est de plus intéressant de noter que pour ces trois cas de rétention légère, le degré de catalase reste comparable à celui des laits normaux; il est cependant maximum dans les deux derniers cas (quartier postérieur gauche de l'animal C. 5 et quartier antérieur droit de l'animal C. 18).

3o Rétention compliquée d'inféction. - Presque tous les laits sont déjà plus ou moins infectés dans la mamelle Si l'expulsion mécanique des germes n'est pas assurée par des traites complètes et régulières, à la rétention vient fatalement s'ajouter l'infection; e'est un fait que, en s'aidant l'un l'autre, ees deux processus évoluent le plus souvent parallèlement. Aussi avons-nous tenu, ces cas étant les plus nombreux, à établir les formules leucocytaires correspondant à cet état; elles figurent au tableau VII, dans la catégorie : rétention compliquée d'infeetion. Examinons-les successivement:

Les échantillons prélevés sur les quartiers antérieur gauche, postérieur gauche et postérieur droit de l'animal C. 18 proviennent d'une mamelle au début de la période de lactation un peu plus d'une semaine après le vêlage; la rétention exagérée de la période colostrale a déterminé une infection légère des trois quartiers précités; seul le quartier antérieur droit est resté sain. Cette infection sournoise, que la clinique n'a pas pu établir, pas plus d'ailleurs qu'un examen superficiel, du lait, seuls la détermination du degré de catalase et l'examen microscopique du lait ont permis de la déceler; le degré de catalase exprimé en 
centimètres cubes d'oxygène dégagé, varie de 2,5 à 4 (chiffres nettement supérieurs à ceux obtenus pour les laits normaux): en outre, à l'examen microscopique, des germes (staphylocoques ou fins bacilles) sont trouvés inclus dans le protoplasma des polynucléaires.

Quelles sont les caractéristiques leucocytaires de ces laits ? Les leucocytes sont abondants dans les frottis, aussi la numération différentielle est-elle beaucoup facilitée. Un pourcentage élevé de polynucléaires variant de 73 à $75 \%$, attire tout d'abord l'attention; ces éléments sont toujours abondants dans le lait, mais, à eux seuls, ils constituent dans les infections, nous le verrons plus loin, les trois quarts et même quelquefois la presque totalité du contenu leucocytaire. Pour les autres catégories de leucocytes, rien ne diffère des autres laits de rétention légère ou prononcée non infectés, à savoir: faible proportion de lymphocytes (1 à $9 \%$ ) au bénéfice des gros mononucléaires ordinaires et de ceux chargés de globules gras, dont la présence est constante dans tous les cas de rétention.

Enfin les deux autres échantillons réunis dans le même groupe (lait du quartier postérieur gauche de l'animal C. 3 et lait du quartier postérieur droit de l'animal C. 10) sont des laits provenant de quartiers infectés objets de traites insuffisantes au troisième et au neuvième mois de la lactation.

En résumé, nous pouvons considérer que LES LAITS DE RÉTENTION SONT TOUJOURS CARACTÉRISÉS PAR LA PRÉSENCE DE GROS MONONUCLÉAIRES CHARgés de globules gras. Plus nombreux que les gros mononucléaires ordinaires dépourvus de matière grasse dans les cas de rétention prononcée, ils sont au contraire moins nombreux qu'eux dans les cas de rétention légère. Ces gros MonONUClÉaIRES LIPOPHAGES NE SONT PAS EXCLUSIVEMENT DES ÉLÉMENTS DU COLOSTRUM, puisque nous les avons rencontrés dans des laits au troisième et au neuvième mois de lactation. Leur présence signifie Rétention, quelle qu'en soit la cause. Aussi, la période colostrale passée, lorsque leur présence sera observée dans le lait, grâce à l'examen leucocytaire que nous préconisons, il sera indiqué de multiplier les traites et de veiller à ce que le pis soit chaque fois complètement vidé. On voit dès maintenant l'intérêt de notre méthode au point de vue économique et au point de vue de la prophylaxie des mammites.

Par ce moyen, en effet, il est permis d'obtenir un rendement maximum que des traites démontrées insuffisantes interdisaient; de plus, la rétention combattue, e'est l'expulsion satisfaisante des germes à chaque traite et la mammite évitée. L'exemple de l'animal C. 5, séjournant dans une étable où sévissait la mammite contagieuse, est tout à fait suggestif à cet égard. Lors d'un premier prélèvement au quatrième mois de la lactation, nous trouvions à l'examen leucocytaire des gros mononucléaires chargés de graisse. Nous étonnant à bon droit de cette cons- 
tatation 4 mois après le vêlage, nous recherchâmes la cause de cètte rétention : l'animal avait eu des gerçures des trayons et, à la suite d'une série de traites douloureuses, était devenu très eraintif; le jeune fils du propriétaire chargé provisoirement de la traite ne s'attachait pas suffisamment à vider complètement le pis de cet animal difficile à traire, "retenant son lait", disait-il. Au deuxième prélèvement, deux mois plus tard, la mamelle était atteinte de mammite streptococcique. $\mathrm{Ne}$ sommes-nous pas en droit de penser que la rétention fut ici une cause favorisante de l'infection?

$4^{\circ}$ Colostrum. - Le colostrum est le premier lait qu'on obtient de la mamelle dans les jours qui suivent immédiatement le part. C'est un liquide jaune, épais, visqueux, collant aux doigts, qui donne, après centrifugation, un gros culot pouvant atteindre 1/5 du volume du lait centrifugé, divisé en deux couches de densité différente : l'une, supérieure, la moins dense, mais la plus volumineuse, albumineuse, gris jaunâtre, l'autre, inférieure, plus réduite, constituée par les éléments cytologiques : cellules épithéliales, leucocytes et hématies. La matière grasse est abondante; elle est fréquemment de couleur jaune safran dans la race normande ; enfin le degré catalasimétrique élevé du colostrum est une connaissance classique.

Quelles sont, au point de vue leucocytaire, les caractéristiques du premier produit de la mamelle?

Comme toutes les fois qu'un appel leucocytaire s'établit dans la glande, qu'il soit causé par la rétention ou l'infection, les leucocytes apparaissent aussitôt très nombreux dans les frottis.

Contrairement à ce qu'on pourrait croire,. les éléments cytologiques, signature de la rétention, n'y sont pas le plus souvent en proportion plus élevée que dans les laits de rétention déjà étudiés; ceci pour la raison que, presque toujours infecté, le colostrum offre dans la majorité des cas une abondante proportion de polynucléaires. Des staphylocoques ont été trouvés phagocytés dans deux des trois cas résumés dans la deuxième partie du tableau VII; seul le quartier postérieur gauche de l'animal N. 6 n'était pas infecté ; le pourcentage des polynucléaires y tombe à 57 , alors que dans le lait staphylococcique des quartiers antérieur gauche du même animal N. 6 et antérieur gauche de l'animal N. 8 , ce pourcentage s'élève à 77 et 92 .

Inversement, les lymphocytes sont plus abondants dans le quartier non infecté que dans les deux autres : $8 \%$ au lieu de $1 \%$. Par ailleurs, nous retrouvons les caractéristiques leucocytaires des laits de rétention prononcée, c'est-à-dire un pourcentage de lipophages supérieur à celui des gros mononucléaires dépourvus de globules gras. Nous devons signaler cependant qu'à la rétention maxima du colostrum correspond une défense plus efficace de l'organisme, car le travail de résorption 
n'est pas gêné par une sécrétion abondante, comme c'est le cas lorsque la rétention s'établit plus tard au cours de la période de lactation. Cette résorption plus poussée se traduit par une phagocytose plus complète des globules gras; aussi les lipophages ont-ils atteint plus généralement le stade miriforme qu'on désigne sous le nom de corpuscule de DonNé. Presque toujours, on observe aussi la phacocytose de petits globules gras par les polynucléaires. En outre, beaucoup de polynucléaires plus ou moins dégénérés offrent un noyau en forme de croissant rejeté en bordure de leur protoplasma dont les fines granulations ont plus ou moins disparu.

L'incorporation maxima de globules gras par les lipophages est une cause de variation importante de la formule leucocytaire, selon que le frottis a été confectionné avec une goutte de colostrum ou avec une goutte de dilution du culot de centrifugation de ce liquide. En effet, sous l'action de la force centrifuge, les lipophages, devenus dans le colostrum de parfaits corpuscules de DonNé, allégés par la graisse dont ils sont bourrés, sont en grande partie entraînés dans la couche de crème superficielle; aussi, leur proportion est-elle toujours plus grande dans les frottis direets, où nous en avons trouvé jusqu'à $42 \%$, que dans les frottis de dilution du culot de centrifugation, où leur proportion n'a jamais dẻpassé $15 \%$.

Retour à la formule leucocytaire normale. - Dans le but d'établir les modifications subies par la formule leucocytaire dans les jours qui suivent le part, nous avons étudié, au Marché de la Villette, le lait du quartier antérieur gauche de l'animál N. 8 pendant les 3 jours consécutifs à son vêlage; l'abatage de l'animal mit fin prématurément à nos investigations que nous aurions voulu poursuivre une bonne semaine ; les résultats acquis sont cependant démonstratifs.

Ce quartier a été vidé eomplètement à la main, après les tétées du veau, plusieurs fois par 24 heures, de façon à éliminer le plus rapidement possible toute rétention. Le premier effet de ces traites répétées fut de faire disparaître au troisième jour toute trace de staphylocoques; aussi le pourcentage des polynucléaires s'abaissa-t-il de 92 à 79 puis à 69 ; par contre, celui des lymphocytes passa de 0,5 à 10 et à 16,5 . Le troisième jour, lipophages et gros mononucléaires sont en proportion égale : $5 \%$; alors que le premier et deuxième jour, le pourcentage des lipophages était légèrement supérieur à celui des gros mononucléaires comme dans le cas de rétention prononcée. (Voir tableau VII, p. 754.)

Il est à présumer que les lipophages auraient diminué progressivement jusqu'à disparition totale dans un temps d'autant plus court que les traites auraient été plus fréquentes et plus poussées.

L'étude du lait du quartier postérieur droit de l'animal N. 10 nous a permis l'observation de la formule leucocytaire d'un lait au septième jour après le vêlage. Il s'agissait d'une vache normande, relevant de fièvre 
vitulaire, dont deux quartiers de la mamelle étaient irrémédiablement perdus pour la lactation à la suite d'une infection grave consécutive à l'insufflation dans des conditions déplorables d'air dans la mamelle. Ces deux quartiers donnaient un liquide roussâtre à forte odeur de nécrose. Le liquide du quartier postérieur droit semblait normal et sa formule leucocytaire fut ainsi établie :

$47 \%$ de polynucléaires,

$44 \%$ de lymphocytes,

$6 \%$ de gros mononucléaires,

$2 \%$ de lipophages et

$1 \%$ de petits mononucléaires.

Nous pouvons donc conclure que la transformation du colostrum en lait normal, sous l'influence des traites ou succions répétées, se traduit dans la formule leucocytaire par la diminution progressive du nombre de polynucléaires et des lipophages et au contraire par l'augmentation du nombre des lymphocytes; à condition, toutefois, que l'infection ordinairement constatée ne s'installe pas définitivement dans la mamelle.

(A suivre.)

\section{BIBLIOGRAPHIE ANALYTIQUE}

\section{LES LIVRES}

Fisk (Walter-W.). - Commercial Casein (Caséine commerciale), 1 vol. de 44 p. + XVI p.relié. The Olsen Publishing Co, Milwaukee (Wisconsin). 1923 (1).

Dans ce petit livre, F. montre quelle est l'importance croissante de la caséine dans l'industrie américaine. La part de la République Argentine est très grande dans les importations américaines.

La caséine, fait-il remarquer, est un des rares produits tirés du lait qui ne soit pas généralement utilisé pour l'alimentation humaine. Il est de fait qu'une très faible portion seulement de la caséine fabriquée est employée pour des fins diététiques. Ce sont les industries des matières plastiques, de la colle et de la papeterie qui sont les gros consommateurs de la caséine.

T. s'étend très largement sur la fabrication de la caséine, mais il emprunte une grande partie de son texte à un travall antérieur de A. O. DAHLBERG : The Manufacture of Casein from Buttermilk of Skim Milk. Bull. 661 du Département d'Agriculture des Etats-Unis 1918.

C'est surtout de la caséine acide dont il parle et il insiste beaucoup sur la prépara-

(1) Nous donnons à la suite l'une de l'autre, trois analyses de livres américains sur la caséine, le premier date de 1923, mais les deux autres sont des livres récents. Nous appelons l'attention de nos lecteurs sur leur grand intérêt. L'industrie de la caseine est étudiée avec le plus grand soin aux Etats-Unis et la meilleure preuve en est dans la publication des livres que nous analysons dans ce numéro (Ch. P.). 